\title{
RENDIMIENTO DE LOS TITULADOS EN PSICOLOGÍA EN LAS PRUEBAS DE ACCESO AL PIR
}

\section{PERFORMANCE OF GRADUATES IN THE INTERNSHIP IN CLINICAL PSYCHOLOGY ENTRANCE EXAMS}

\author{
Eva EXPÓSITO-CASAS ${ }^{1}$, ANA GONZÁLEZ-BENITO ${ }^{1}$, \\ ESTHER LÓPEZ-MARTÍN ${ }^{1}$ Y ALEXIS MORENO-PULIDO ${ }^{1}$
}

Cómo referenciar este artículo/How to reference this article:

Expósito-Casas, E., González-Benito, A., López-Martín, E. y Moreno-Pulido, A. (2016). Rendi mi ento de los titulados en Psicología en las pruebas de acceso al PIR [Performance of graduates in the Internship in Clinical Psychology entrance exams]. Acción Psicológica, 13(1), 145-158. http://dx.doi.org/10.5944/ap.13.1.17429

\begin{abstract}
Resumen
Entre las titulaciones más demandadas por parte de los futuros estudiantes universitarios, figura el Grado en Psicología. Este interés ha traído consigo un incremento de la oferta de plazas en los últimos años, tanto en universidades públicas como privadas, $\mathrm{y}$, consecuentemente, un aumento del número de egresados con dicho perfil académico/profesional. Dentro de las posibles salidas profesionales de estos titulados, se encuentra el acceso al Sistema Nacional de Salud. Para ello, los candidatos deben superar previamente las pruebas selectivas de acceso a plazas de formación
\end{abstract}

sanitaria especializada, tras lo cual, aquellos que obtienen plaza, se incorporan al sistema de formación de posgrado en régimen de residencia (Psicólogo Interno Residente PIR-). El presente trabajo analiza el rendimiento de los egresados de las distintas Facultades de Psicología españolas en las pruebas de acceso al Sistema Nacional de Salud durante el periodo 2013-2016, y las posibles diferencias en función de variables como el tamaño o la titularidad de las universidades. El carácter nacional de la convocatoria y la objetividad de las pruebas, hacen posible evaluar el impacto educativo y laboral de estas instituciones, incorporando al enfoque de la rendición de cuentas una dimensión social.

Correspondencia: Eva Expósito-Casas. Departamento de Métodos de Investigación y Diagnóstico en Educación II. Facultad de Educación, Universidad Nacional de Educación a Distancia (UNED).

Email: evaexpositocasas@edu.uned.es

${ }^{1}$ Universidad Nacional de Educación a Distancia (UNED), España.

Recibido: 23 de marzo de 2016.

Aceptado: 12 de mayo de 2016. 
Palabras clave: universidades españolas; impacto educativo; rendimiento académico; estudios universitarios; Psicólogo Interno Residente; responsabilidad social.

\begin{abstract}
The university degree in Psychology is one of the most popular among prospective university students. As a result, Spanish public and private universities have increased the amount of places and the number of university graduates in Psychology. Access to the National Health System is one professional possibility for these graduates. Applicants must pass an exam to obtain an Internship in Clinical Psychology at the National Health System. This paper analyses the graduate's exam performance during the period 2013-2016, and also possible differences depending on the size or the ownership of their universities of origin. The objectivity of the national call makes it possible to evaluate the educational and labor impact of universities in society. This perspective incorporates a social dimension to the accountability approach.
\end{abstract}

Keywords: Spanish universities; impact of education; academic achievement; university studies; Internship in Clinical Psychology; social responsibility.

\section{Introducción}

De acuerdo con las estadísticas de Universidades Centros y Titulaciones, elaboradas por el Ministerio de Educación, Cultura y Deporte (MECyD), la titulación de Psicología presenta una alta demanda. Así, en el curso académico 2015-2016, el porcentaje de preferencia por la titulación (tasa de solicitud del título en primera opción) se situó en el $250 \%$, a nivel nacional, alcanzando un valor máximo del $680 \%$ en la comunidad autónoma de Extremadura. Asimismo, en dicho curso, la nota me- dia para acceder a la titulación fue de 7.78 puntos, ofertándose más de 5000 plazas. Estas cifras ponen de manifiesto que la solicitud de estos estudios supera considerablemente la oferta de las universidades.

En respuesta al interés por la titulación, se ha producido un incremento de la oferta formativa, fundamentalmente por parte de las universidades privadas, con el consiguiente crecimiento del número de egresados con este perfil académico/profesional (González-Bach, 2015). Sin embargo, la atención de las universidades a la demanda de los estudiantes, parece no estar en equilibrio con las necesidades del mercado laboral. De esta forma, el MECyD y la Conferencia de Consejos Sociales (2014), informan de que, en el año 2014, la tasa de afiliación a la Seguridad Social de los estudiantes en Psicología que se graduaron en el curso académico 2009-2010, era del $60.8 \%$, es decir, aproximadamente cuatro puntos porcentuales por debajo de la media nacional para el conjunto de las titulaciones (64.4\%), y muy alejada del porcentaje relativo a otras profesiones sanitarias como Medicina (92.9 \%) y Farmacia (81.4\%).

Los problemas de ocupación de los especialistas en Psicología contrastan con las necesidades de atención psicológica por parte de la población; tal y como señala la Organización Mundial de la Salud (2014), para mejorar los servicios de salud mental se deben superar algunos obstáculos, entre los que se encuentra la escasez de recursos humanos. En el mismo sentido se pronuncia el Consejo General de la Psicología (COP), que manifiesta que en España la atención primaria cuenta con 4.3 psicólogos por cada 100000 habitantes, muy por debajo de la media europea que se sitúa en 18 psicólogos por cada 100000 habitantes (COP, 2015).

En España, la creación del título de Psicólogo Especialista en Psicología Clínica, regulado por el Real Decreto $2490 / 1998^{2}$, dio respuesta a la voluntad de atender las necesidades del sistema sanitario en el ámbito de

\footnotetext{
2 Derogado por el Real Decreto $183 / 2008$, de 8 de febrero, por el que se determinan y clasifican las especialidades en ciencias de la salud y se desarrollan determinados aspectos del sistema de formación sanitaria especializada.
} 
la salud mental. El sistema de obtención de este título, emula el establecido para los títulos de Médico y Farmacéutico Especialista, que persiguen asegurar:

el alto nivel profesional de quienes desarrollan su actividad en el ámbito de la atención sanitaria y, con ello, un elevado índice de calidad de los centros, servicios y profesionales a los que corresponde hacer efectivo el derecho a la protección de la salud que proclama el artículo 43 de la constitución (Real Decreto 2490/1998 pp. 39538-39539).

Los titulados en Psicología que aspiren a obtener el título de Psicólogo Especialista en Psicología Clínica deben concurrir a las convocatorias de las pruebas selectivas reguladas por el artículo 22.1 de la Ley 44/2003, de 21 de noviembre, de ordenación de las profesiones sanitarias. En esta normativa se establece que el "acceso a la formación sanitaria especializada se efectuará a través de una convocatoria anual de carácter nacional" (BOE núm. 280, 22 de noviembre de 2003, p. 41450). A su vez, en el apartado 2 del citado artículo, modificado por la disposición adicional 2.1 del Real Decreto-Ley 9/2011, de 19 de agosto, se indica que será el Ministerio de Sanidad, Política Social e Igualdad, previo informe del Ministerio de Educación y de la Comisión de Recursos Humanos del Sistema Nacional de Salud (SNS), el organismo encargado de establecer las normas que regulen la convocatoria anual "que consistirá en una prueba o conjunto de pruebas, que evaluarán conocimientos teóricos, prácticos $\mathrm{y}$, en su caso, habilidades clínicas, comunicativas y méritos académicos y profesionales de los aspirantes" (BOE núm. 200, 20 de agosto de 2011, p. 93164). Los candidatos que obtienen una plaza en el sistema de formación de posgrado en régimen de residencia (Psicólogo Interno Residente -PIR-), conseguirán el título al finalizar satisfactoriamente este periodo de formación (Carrobles, 2012).

Tal y como puede observarse, el SNS proporciona una salida profesional a los egresados universitarios del campo de la Psicología Clínica y, a su vez, las universidades ven cumplidas sus misiones educadora y social a través de su contribución a dicho sistema. Esta aportación de las instituciones de educación superior se enmarca dentro del contexto de la rendición de cuentas.
La rendición de cuentas lleva implícito un compromiso de responsabilidad de cualquier organización hacia la sociedad en la que residen sus objetivos, e implica transparencia en la información relativa a la consecución de los fines marcados y en la forma en que se ha conseguido ese cumplimiento (CRUE, 2005). A su vez, la rendición de cuentas se incluye dentro de la responsabilidad social de las universidades (RSU) y de la necesidad de que las universidades informen del impacto de sus actividades, a fin de poner en valor su legitimidad social frente a la comunidad (Dill, 2001; Garde Sánchez, Rodríguez Bolívar y López-Hernández, 2013; Larrán-Jorge y Andrades-Peña, 2015; Pacenza y Silva, 2013; Ramallo, 2015).

En el ámbito de este trabajo, los resultados obtenidos por los estudiantes de Psicología que se presentan a las pruebas de acceso al SNS, se considera un indicador de la calidad de la enseñanza recibida y del impacto de esta formación en la sociedad. Por ello, en el presente estudio se analiza el rendimiento de los titulados de las distintas universidades españolas que imparten el Grado en Psicología en las pruebas selectivas de acceso a la formación sanitaria especializada del SNS. Asimismo, este trabajo evalúa el impacto educativo y laboral de tales universidades, incorporando al enfoque de la rendición de cuentas una dimensión social. Cabe señalar que la RSU de las instituciones analizadas, no se limita a la contribución relativa al SNS, pues las dimensiones en las que se podría estructurar dicha responsabilidad social abarcarían un espectro mucho mayor, que excede los objetivos de esta propuesta. No obstante, desde esta contribución, se plantea la utilización de una variable de gran utilidad a tales efectos: los resultados en las pruebas de selección de candidatos a ocupar plazas de Psicólogo Interno Residente (PIR), cuyo carácter objetivo, periódico y global, vinculado estrechamente con una función social, pone de manifiesto su posible utilidad informativa.

\section{Método}

El diseño de este estudio es de carácter cuantitativo, no experimental, con una aproximación descriptiva a datos secundarios procedentes del Ministerio de Sanidad, 
Servicios Sociales e Igualdad. Estos datos hacen referencia al número de estudiantes que han superado la nota de corte en las pruebas de selección de candidatos a ocupar plazas de Psicólogo Interno Residente (PIR) en las convocatorias 2013-2014, 2014-2015 y 2015-2016, así como el total de candidatos presentados a examen en las convocatorias mencionadas.

\section{Población y muestra}

La población objeto de estudio está constituida por los 10107 estudiantes que se han presentado a las pruebas referidas en el periodo objeto de estudio. En la Tabla 1 se muestran dichos sujetos agrupados de acuerdo con la universidad de origen.

En total se contemplan 40 universidades públicas y privadas, que ofertan la titulación de Psicología en la modalidad presencial y a distancia. El resto de universidades que imparten estos estudios en España (ocho de titularidad privada y una de titularidad pública) no han sido consideradas, ya que ningún estudiante de estas instituciones se ha presentado a las pruebas selectivas para el acceso al SNS.

\section{Variables}

La puntuación en la prueba selectiva, se estima a partir de la respuesta de los candidatos a una prueba de opción múltiple compuesta por 225 reactivos (más 10 preguntas de reserva) y limitada a 5 horas de duración, características comunes a las tres convocatorias analizadas (Orden SSI/1695/2013, de 12 de septiembre;
Orden SSI/1674/2014, de 10 de septiembre; Orden SSI/1892/2015, de 10 de septiembre).

La prueba de elección múltiple, se considera superada para aquellos aspirantes que obtengan una valoración particular igual o superior a un $35 \%$ de la media aritmética de las diez mejores puntuaciones particulares del ejercicio, considerándose de manera exclusiva los números enteros resultado de esta operación (Orden SSI/1695/2013, de 12 de septiembre; Orden SSI/1674/2014, de 10 de septiembre; Orden SSI/1892/2015, de 10 de septiembre).

Por último, en cuanto al cálculo de la puntuación global en la prueba, cabe destacar que los criterios establecidos son los siguientes:

Primera.-Cada contestación válida recibirá una valoración de tres puntos, se restará un punto por cada una de las respuestas incorrectas, se dejarán sin valorar las preguntas no respondidas y de las operaciones anteriores se obtendrá la valoración particular del ejercicio realizado por cada aspirante.

Segunda.-Evaluados todos los ejercicios, se hallará la media aritmética de las diez máximas valoraciones particulares obtenidas y a esta media aritmética le corresponderán 90 puntos.

Tercera.-La puntuación final de este ejercicio se obtendrá para cada aspirante multiplicando por 90 la valoración particular del mismo y dividiendo el producto por la media aritmética a que se refiere la anterior regla segunda. Esta puntuación final se expresará con los primeros cuatro decimales obtenidos, despreciándose el resto (Orden SSI/1892/2015, de 10 de septiembre).

Tabla 1

Distribución de los sujetos que forman parte de la muestra en función de la titularidad y la modalidad de enseñanza de la universidad de origen

\begin{tabular}{lcccc}
\hline & \multicolumn{2}{c}{ Universidades Públicas } & \multicolumn{2}{c}{ Universidades Privadas } \\
\cline { 2 - 5 } & Presentados & Instituciones & Presentados & Instituciones \\
\hline Presencial & $7790(84.70 \%)$ & $26(96.30 \%)$ & $812(89.23 \%)$ & $12(92.31 \%)$ \\
No presencial & $1407(15.30 \%)$ & $1(3.70 \%)$ & $98(10.77 \%)$ & $1(7.69 \%)$ \\
\hline
\end{tabular}


De manera complementaria, se ha incluido el número de titulados que finalmente obtienen plaza en el programa de formación PIR.

En relación a las variables de caracterización de las universidades, se han considerado las siguientes: (a) Titularidad (Pública o Privada), (b) Tamaño (a partir del número de estudiantes matriculados en la titulación de Psicología en las diferentes instituciones durante el curso académico $2014-2015^{3}$, éstas se han clasificado en Grandes $-50 \%$ de universidades con mayor número de alumnos matriculados- y Pequeñas $-50 \%$ de universidades con menor número de alumnos matriculados-), y (c) Modalidad (Presencial o No presencial).

\section{Resultados}

\section{Evolución del número de titulados en Psicología por universidad que se presentan y superan las pruebas selectivas para el acceso al SNS}

La Tabla 2 muestra el número de titulados en Psicología por universidad que se han presentado a las pruebas de acceso al SNS en cada una de las tres convocatorias analizadas, así como el número de aspirantes que han superado dichas pruebas. Tal y como puede observarse, durante el periodo considerado, se han presentado a las pruebas un total de 10107 sujetos, de los cuales han superado la nota de corte 8563. Teniendo en cuenta que los aspirantes proceden de 40 universidades españolas, el promedio de titulados por universidad que se presentan y superan dichas pruebas sería de 253 y 214 sujetos, respectivamente.

En cualquier caso, resulta destacable la enorme variabilidad en el número de aspirantes que proceden de unas y otras instituciones de educación superior. En este sentido, se observa cómo el número de sujetos que se presentan a las diferentes convocatorias por universidad oscila entre 1 (convocatorias 2013-2014 y 2015-2016) y 478 (convocatoria 2013-2015). En el caso de los sujetos que superan las pruebas por universidad, los valores mínimos y máximos observados son 0 (convocatoria 2013-2014) y 408 (convocatorias 2014-2015 y 20152016).

El análisis de la evolución de los sujetos presentados a las tres convocatorias, muestra cómo las posibles diferencias en el número de titulados presentados no resultan estadísticamente significativas (Chi-cuadrado de Fried$\operatorname{man}=3.279$, g.1. $=2, p=.194)$. Por el contrario, sí existen diferencias significativas en el número de sujetos

Tabla 2

Número de sujetos por universidad que se presentan a las pruebas de acceso al SNS y que superan dichas pruebas. Análisis descriptivo y de normalidad

\begin{tabular}{|c|c|c|c|c|c|c|c|c|}
\hline & \multicolumn{4}{|c|}{ Presentados } & \multicolumn{4}{|c|}{ Superan } \\
\hline & $2013-14$ & $2014-15$ & $2015-16$ & TOTAL & 2013-14 & $2014-15$ & $2015-16$ & TOTAL \\
\hline N (Universidades) & 37 & 36 & 40 & 40 & 37 & 36 & 40 & 40 \\
\hline Suma (Total aspirantes) & 3345 & 3427 & 3335 & 10107 & 2633 & 2949 & 2981 & 8563 \\
\hline Mínimo & 1 & 2 & 1 & 1 & 0 & 2 & 1 & 1 \\
\hline Máximo & 478 & 476 & 453 & 1407 & 358 & 408 & 408 & 1174 \\
\hline Media & 90 & 95 & 83 & 253 & 71 & 82 & 75 & 214 \\
\hline Mediana & 50 & 59 & 53 & 146 & 40 & 50 & 44 & 119 \\
\hline Moda & 4 & 5 & 2 & 405 & 3 & 4 & 1 & 1 \\
\hline Desviación estándar & 105 & 105 & 97 & 304 & 82 & 91 & 89 & 259 \\
\hline Kolmogorov-Smirnov & $.196^{* *}$ & $.188^{* *}$ & $.199^{* *}$ & $.204^{\star *}$ & $.192^{* *}$ & $.198^{\star *}$ & $.204^{\star *}$ & $.205^{\star *}$ \\
\hline
\end{tabular}


Tabla 3

Porcentaje de sujetos que aprueban la prueba de acceso al SNS. Análisis descriptivo y de normalidad

\begin{tabular}{lrrrr}
\hline & $\% 2013-2014$ & $\% 2014-2015$ & $\% 2015-2016$ & $\%$ Total \\
\hline N (Universidades) & 37 & 36 & 40 & 40 \\
Mínimo & $0.0 \%$ & $55.0 \%$ & $50.0 \%$ & $33.3 \%$ \\
Máximo & $100.0 \%$ & $100.0 \%$ & $100.0 \%$ & $100.0 \%$ \\
Media & $75.7 \%$ & $85.3 \%$ & $84.7 \%$ & $81.7 \%$ \\
Mediana & $80.0 \%$ & $86.5 \%$ & $88.0 \%$ & $84.5 \%$ \\
Moda & $75.0 \%$ & $85.0 \%$ & $100.0 \%$ & $90.0 \%$ \\
Desviación estándar & $18.4 \%$ & $10.3 \%$ & $13.28 \%$ & $12.72 \%$ \\
Kolmogorov-Smirnov & $.246^{* *}$ & $.155^{*}$ & $.203^{* *}$ & $.227^{* *}$ \\
\hline
\end{tabular}

Nota. ${ }^{* \star}$ La variable analizada no se distribuye normalmente a un nivel de confianza del $99 \%(\alpha=0.01)$.

* La variable analizada no se distribuye normalmente a un nivel de confianza del $95 \%(\alpha=0.05)$.

que aprueban las pruebas de acceso al SNS en dichas convocatorias (Chi-cuadrado de Friedman $=16.504$, g.l. $=2, p=.000)$. Así, el número de aspirantes que superan la nota media de corte resulta menor en la convocatoria 2013-2014 que en las convocatorias 2014-2015 ( $Z$ de Wilcoxon $=-3.710, p=.000)$ y 2015-2016 (Z de Wilcoxon $=-3.427, p=.289$ ), no apreciándose diferencias en los resultados de estas dos últimas convocatorias ( $\mathrm{Z}$ de Wilcoxon $=-1.060, p=.289$ ).

\section{Porcentaje de sujetos que aprueban la prueba de acceso al SNS por universidad y análisis de las diferencias en función del tamaño y de la titularidad de las universidades}

El porcentaje de estudiantes de cada una de las universidades que superan las pruebas de acceso, respecto al número total de titulados de estas instituciones que se presentan al examen, se recoge en la Tabla 3. Como puede observarse, en general, el porcentaje de sujetos que superan la prueba de acceso es superior al $75 \%$ en las tres convocatorias. No obstante, al igual que se apuntaba en el apartado anterior, la variabilidad de los resultados obtenidos por los titulados procedentes de las distintas universidades es importante.

Los diagramas de cajas plasmados en la Figura 1, informan del porcentaje de sujetos de las diferentes universidades que superan las pruebas de acceso en cada convocatoria, y en el conjunto del periodo considerado, en función de la titularidad de dichas instituciones. La información representada muestra cómo en todos los casos, la distribución de los porcentajes de aprobados obtenidos por las instituciones públicas, se sitúa por encima de la distribución de porcentajes de las universidades privadas. A su vez, en el caso de las universidades públicas la variabilidad en los resultados obtenidos es menor.

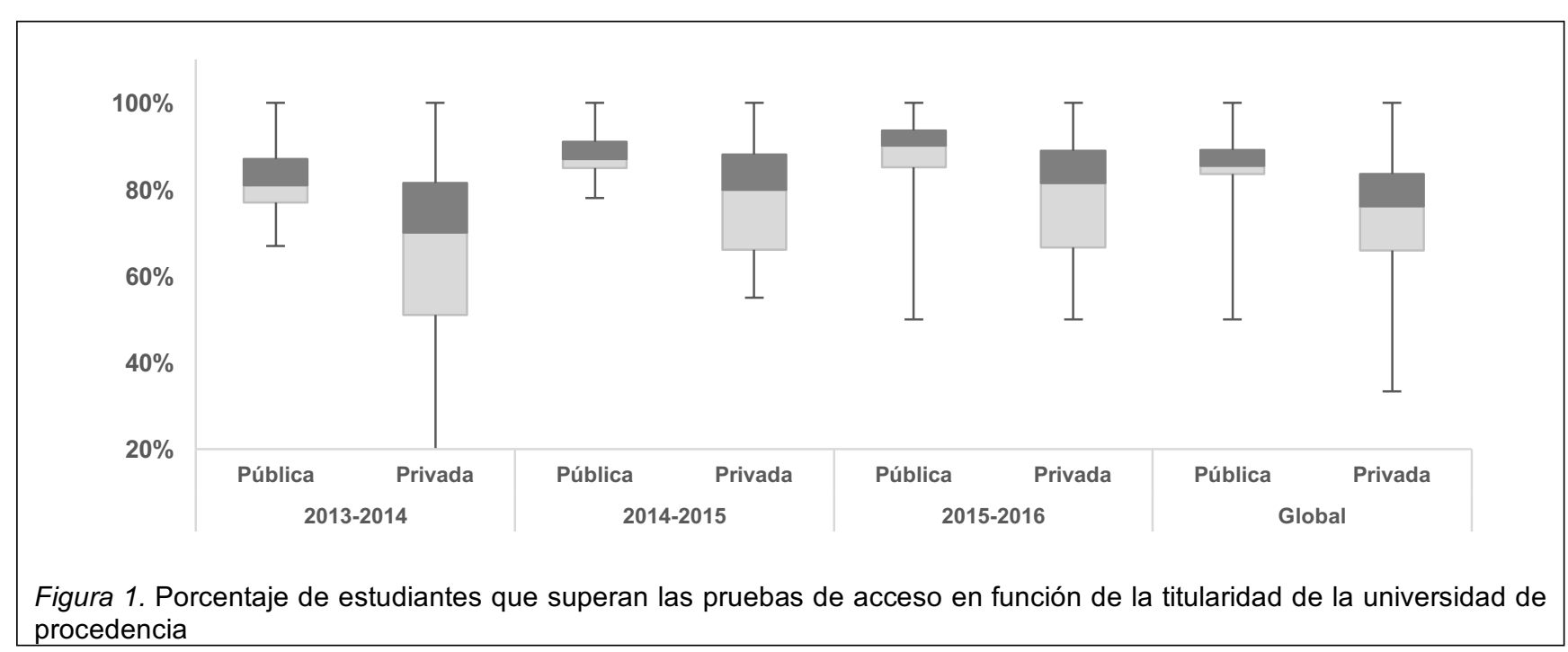




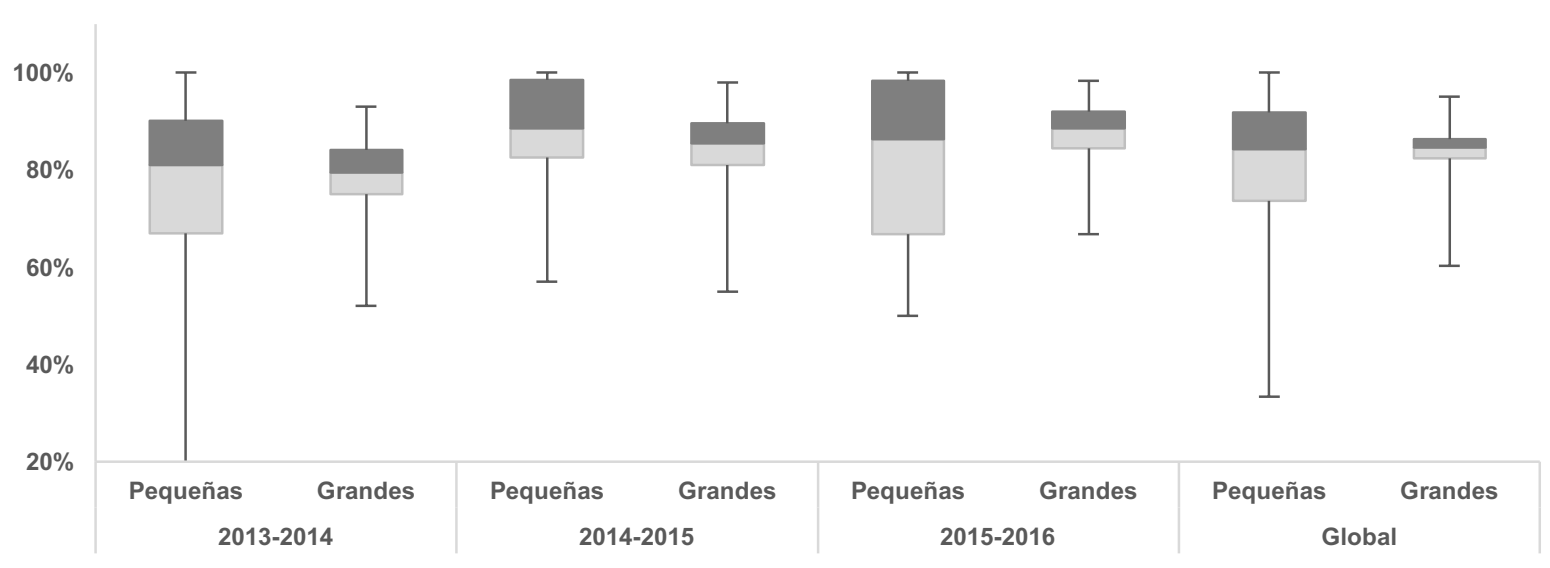

Figura 2. Porcentaje de estudiantes que superan las pruebas de acceso en función del tamaño de la universidad de procedencia

El análisis de la significatividad de las diferencias observadas, llevado a cabo a través de la prueba $U$ de Mann-Whitney, confirma los mejores resultados de los estudiantes procedentes de universidades públicas en las convocatorias 2013-2014 (U de Mann-Withney $=70.5$, $p=.016$ ) y 2014-2015 (U de Mann-Withney $=76.0$, $p=.035)$, así como en el conjunto del periodo contemplado ( $\mathrm{U}$ de Mann-Withney $=82.0, p=.006)$. Por su parte, en la convocatoria 2015-2016, el porcentaje de aprobados de los sujetos procedentes de uno y otro tipo de instituciones no difiere de manera significativa ( $U$ de Mann-Withney $=119.0, p=.106$ ).

Atendiendo al tamaño de las instituciones de educación superior, la Figura 2 representa el porcentaje de titulados de las diferentes instituciones que superan las pruebas, diferenciando los resultados de las universidades grandes y pequeñas. La representación de la distribución de porcentajes de aprobados para los diferentes subgrupos, informa de una mayor variabilidad de los resultados obtenidos por las universidades pequeñas. En cualquier caso, el análisis de las diferencias en los porcentajes de sujetos que superan las pruebas de acceso al SNS en función del tamaño de las instituciones, muestra cómo en las tres convocatorias consideradas: 2013-2015 (U de Mann-Withney $=149.5, p=.838$ ), 2014-2015 (U de Mann-Withney=103.0, $p=.177$ ) y 2015-2016 (U de Mann-Withney =172.0, $p=.594$ ), dichas diferencias no resultan estadísticamente significativas. La superioridad en los resultados de un

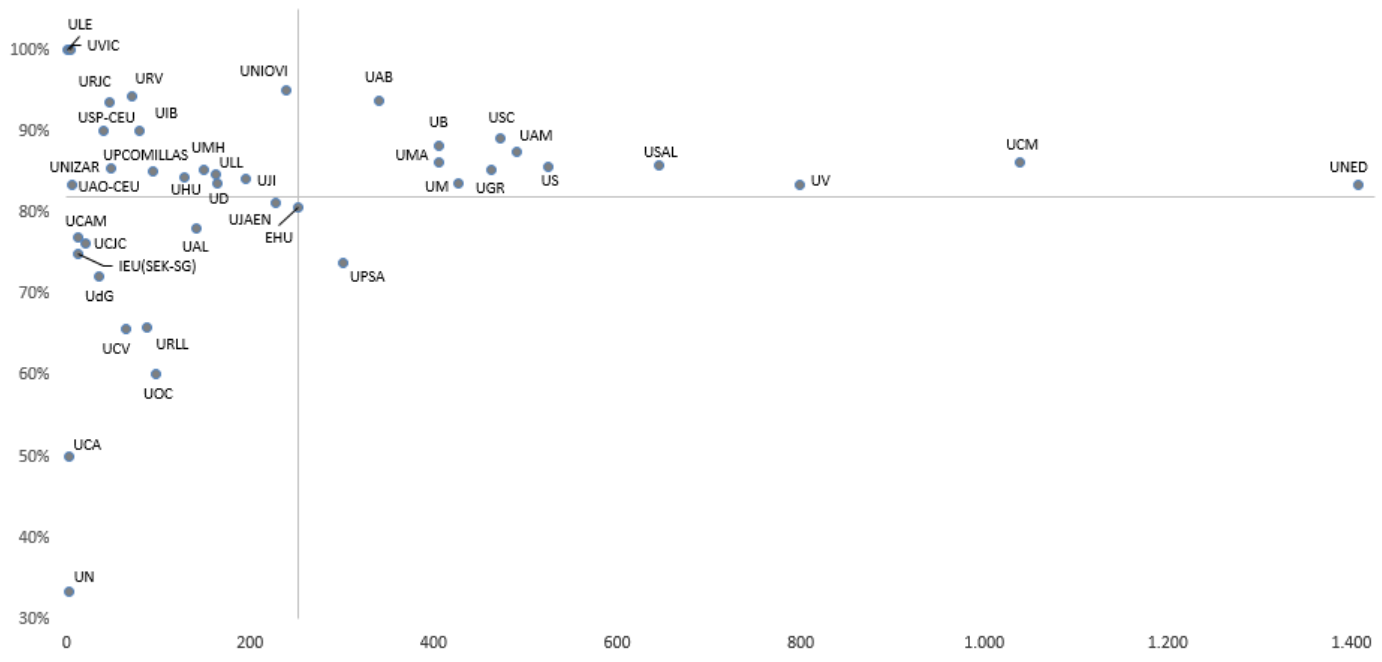

Figura 3. Distribución del número de sujetos presentados y porcentaje de aprobados por universidad 


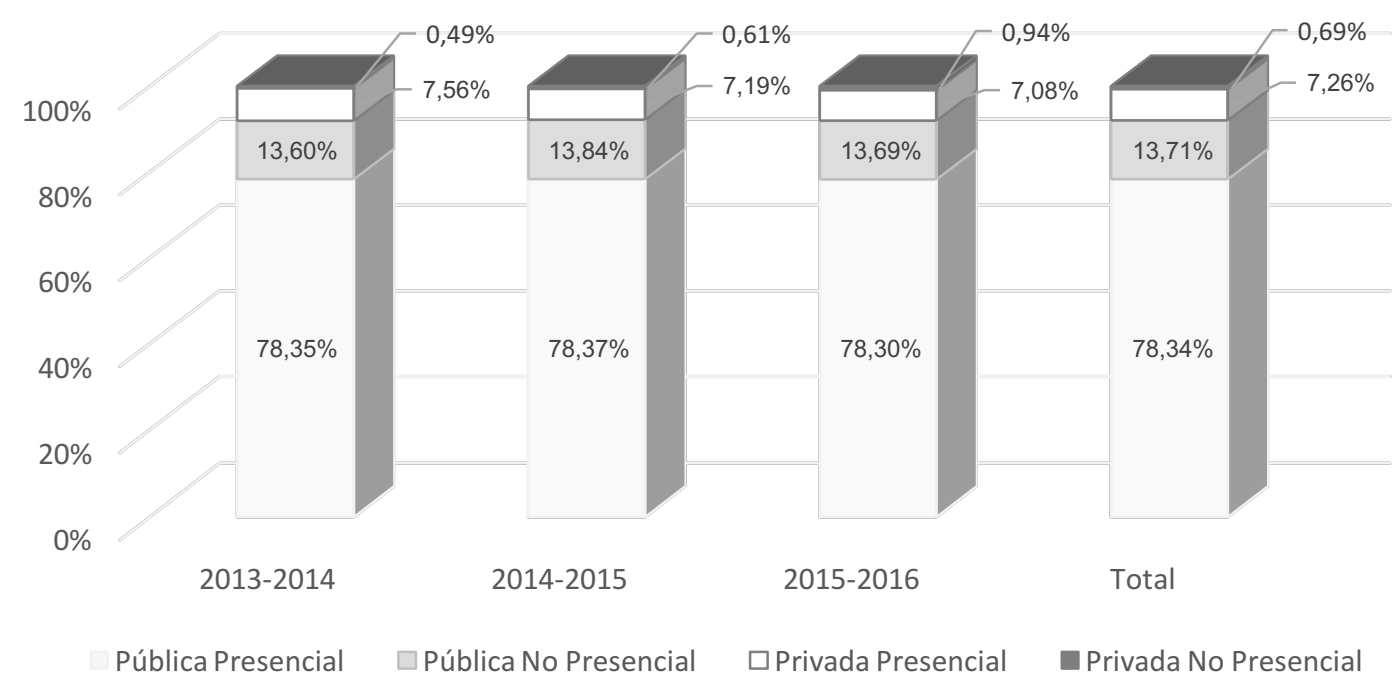

Figura 4. Rendimiento en las pruebas selectivas por universidad de procedencia

tipo de universidad frente a otro, tampoco se pone de manifiesto al considerar la totalidad del periodo contemplado (U de Mann-Withney $=183.0, p=.817$ ).

La dispersión observada en la figura anterior, también se percibe al contemplar la Figura 3. En dicha figura se representa el número de sujetos presentados (x) y el porcentaje de aprobados de las distintas universidades (y). La figura se divide en cuatro cuadrantes, atendiendo al promedio de las dos variables representadas. De esta forma, en el cuadrante I se ubican las 12 universidades cuyos estudiantes se sitúan por encima de la media tanto en el número de presentados como de aprobados. Destaca el hecho de que en todos los casos, se trate de universidades de titularidad pública.

En los cuadrantes II y III se localizan las universidades que se posicionan por debajo de la media en el número de estudiantes presentados y que, respectivamente, obtienen unos resultados por encima y por debajo de la media en cuanto a las tasas de aprobados. Finalmente, en el cuadrante IV, se encuentra una institución que, aunque presenta un número de estudiantes por encima de la media, sus resultados son ligeramente inferiores al porcentaje medio de aprobados.

\section{Rendimiento y contribución de las universidades al SNS}

Centrando el análisis en el rendimiento que obtienen las universidades en las pruebas selectivas, la Figura 4 representa el rendimiento global de las universidades públicas, privadas, presenciales y no presenciales en las pruebas referidas. Del total de sujetos que han superado la nota de corte en las distintas convocatorias, un $78.34 \%$ proceden de universidades públicas presenciales, un $13.71 \%$ de la universidad pública no presencial, un $7.26 \%$ del conjunto de universidades privadas presenciales y, finalmente, un $0.69 \%$ de la universidad privada no presencial que forma parte de este estudio.

Para concluir este apartado, se ha analizado la contribución de las distintas universidades al SNS, y por añadidura, al conjunto de la sociedad, mediante el estudio del número de titulados que obtienen plaza en el 


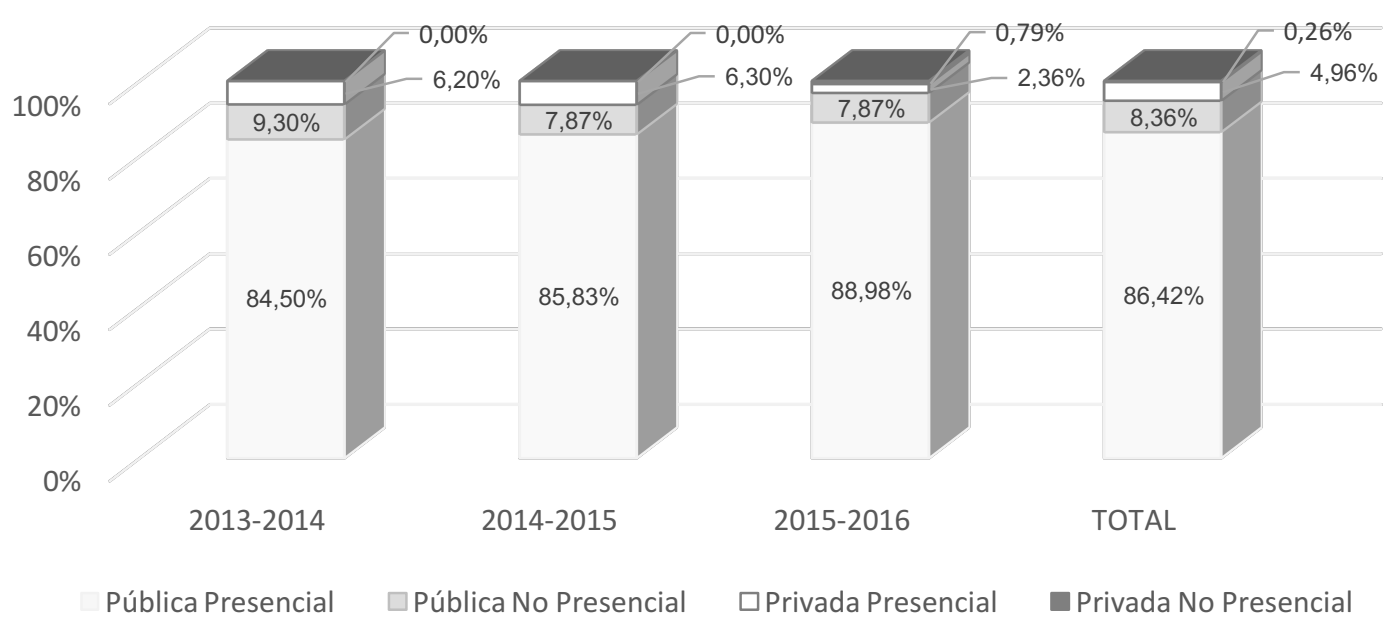

Figura 5. Distribución de plazas por universidad de procedencia

programa de formación PIR. La Figura 5, representa la distribución de estos sujetos en función de la universidad de procedencia.

En sintonía con los datos analizados con anterioridad, el gráfico informa de una importante contribución de las universidades públicas presenciales $(86.42 \%$ del total del periodo) y de la universidad pública no presencial ( $8.36 \%$ del total del periodo) al número de titulados que obtienen plaza en el programa de formación PIR, poniendo de manifiesto la destacada aportación social de estas universidades.

\section{Conclusiones}

El objetivo del presente trabajo ha sido analizar el rendimiento de los egresados de las distintas universidades españolas que imparten la titulación de Psicología en las pruebas selectivas de acceso al PIR. Esta evaluación del impacto educativo y laboral de las universidades, supone incorporar al enfoque de la rendición de cuentas una dimensión social.

La alta demanda de los estudios de Psicología en España durante los últimos años, ha venido acompañada de un incremento de la oferta formativa (especialmente en universidades privadas) y, en consecuencia, del número de egresados con dicho perfil académico/profesional.

Una vía importante de desarrollo profesional para estos egresados, pasa por su especialización como Psicólogos Clínicos. Para ello, los aspirantes deben superar las pruebas selectivas establecidas por la Ley de ordenación de las profesiones sanitarias (Ley 44/2003, de 21 de noviembre). A la luz de los datos procedentes de las tres últimas convocatorias (2013-2014, 2014-2015 y 20152016), se observa cómo se trata de una especialización muy demandada, siendo el número de aspirantes en cada una de las convocatorias superior a 3000 .

Este trabajo evidencia la importante variabilidad en los resultados obtenidos por los egresados procedentes de las distintas universidades. A este respecto, la titularidad de las universidades puede considerarse una de las variables que permiten explicar dichas diferencias. Así, en las tres convocatorias analizadas, el porcentaje de estudiantes procedentes de universidades públicas que superan el ejercicio fue superior al porcentaje de los titulados en centros privados. Tales diferencias han resultado ser estadísticamente significativas para las convocatorias 2013/2014 y 2014/2015, así como en el conjunto del periodo. En cuanto al tamaño del centro, las diferencias en- 
contradas no resultan significativas, aunque cabe destacar una mayor dispersión en los resultados obtenidos por las universidades de menor tamaño.

El estudio llevado a cabo también ha permitido identificar las 12 instituciones de las cuales proceden un número de aspirantes superior a la media y que, además, sus egresados tienden a rendir por encima del promedio en las pruebas de acceso al PIR. Estas universidades, podrían ser identificadas como universidades de alto impacto social, pues la calidad de la enseñanza que reciben sus estudiantes, parece traducirse en un mejor desempeño en las mencionadas pruebas selectivas.

En cuanto al número de titulados que obtienen plaza en el programa de formación PIR, el porcentaje de estudiantes procedentes de universidades públicas, que finalmente obtienen plaza, es superior al $90 \%$ en las tres convocatorias objeto de estudio, alcanzando un $92.05 \%$ como valor total de todo el periodo. De esta forma, se puede destacar la notable contribución social de los centros universitarios de carácter público al SNS.

A pesar del valor informativo de este estudio, resulta importante hacer referencia a algunas de las principales limitaciones derivadas de los datos disponibles. De un lado, hubiese sido interesante analizar el porcentaje de sujetos presentados a estas pruebas en relación con el número total de titulados de las universidades. Por otro lado, no se ha tomado en consideración la formación que hayan podido recibir los egresados para la preparación de estas pruebas selectivas y su presumible influencia en la superación de las mismas.

\section{Referencias}

Carrobles, J. A. (2012). Psicólogo clínico y/o psicólogo general sanitario, Behavioral Psychology/ Psicología Conductual, 20(2), 449-470.

Casilla, D. y Camacho, H. (2012). Evaluación de la Responsabilidad Social Universitaria, Opción, 28(69), 452-465. Recuperado de http://www.redalyc.org/pdf/310/31025702004.pdf
Consejo General de la Psicología (2015). Los Psicólogos Clínicos reclaman su presencia en los centros de Atención Primaria. Recuperado de http://www.cop.es/infocop/pdf/2651.pdf

CRUE (2005). La rendicion de cuentas (accountability). Boletín Electrónico, 28. Recuperado de ima.uni.edu.pe/fweb05/dic05/cuentas.doc.

Garde Sánchez, R., Rodríguez Bolívar, M. y LópezHernández, A. M. (2013). Online Disclosure of University Social Responsibility: A Comparative Study of Public and Private US Universities, Environmental Education Research, 19(6), 709746.

http://dx.doi.org/10.1080/13504622.2012.749976

Dill, D. D. (2001). The regulation of public research universities: changes in academic competition and implications for university autonomy and accountability. Higher Education Policy, 14(1), 2135.

González-Blanch, C. (2015). La psicología clínica después de la psicología general sanitaria en España: una propuesta razonada. Papeles del Psicólogo, 36(1), 9-18. Recuperado de http://www.papelesdelpsicologo.es/pdf/2485.pdf

Larrán-Jorge, M. y Andrades-Peña, F. J. (2015). Análisis de la responsabilidad social universitaria desde diferentes enfoques teóricos, Revista Iberoamericana de Educación Superior, 6(15), 91$107 . \quad \mathrm{http}: / / \mathrm{dx}$.doi.org/10.1016/S20072872(15)30005-6

Ley 44/2003, de 21 de noviembre, de ordenación de las profesiones sanitarias. Boletín Oficial del Estado, 280, 41442-41458. Recuperado de https://www.boe.es/buscar/act.php?id=BOE-A2003-21340

Ministerio de Educación, Cultura y Deporte. Estadística de universidades, centros y titulaciones. Curso 2015-2016. Recuperado de http://www.mecd.gob.es/educacion-mecd/areas- 
educacion/universidades/estadisticas-

informes/estadisticas/universidades-centros-

titulaciones/curso-2015-2016.html

Ministerio de Educación, Cultura y Deporte y Conferencia de Consejos Sociales (2014). Inserción laboral de los egresados universitarios. La perspectiva de la afiliación a la Seguridad Social 2014. Recuperado de http://ccsu.es/content/inserci\%C3\%B3n-laboral-delos-egresados-universitarios-la-perspectiva-de-laafiliaci $\% \mathrm{C} 3 \% \mathrm{~B} 3 n-\mathrm{la}$

Orden SSI/1695/2013, de 12 de septiembre, por la que se aprueba la convocatoria de pruebas selectivas 2013 para el acceso en el año 2014, a plazas de formación sanitaria especializada para Médicos, Farmacéuticos y otros graduados/licenciados universitarios del ámbito de la Psicología, la Química, la Biología y la Física. Boletín Oficial del Estado, 228, 76302-76538. Recuperado de: https://www.boe.es/diario_boe/txt.php?id=BOE-A2013-9826

Orden SSI/1674/2014, de 10 de septiembre, por la que se aprueba la oferta de plazas y la convocatoria de pruebas selectivas 2014 para el acceso en el año 2015, a plazas de formación sanitaria especializada para Médicos, Farmacéuticos, Enfermeros y otros graduados/licenciados universitarios del ámbito de la Psicología, la Química, la Biología y la Física. Boletín Oficial del Estado, 226, 72679-72956. Recuperado de: https://www.boe.es/diario_boe/txt.php?id=BOE-A2014-9474

Orden SSI/1892/2015, de 10 de septiembre, por la que se aprueba la oferta de plazas y la convocatoria de pruebas selectivas 2015 para el acceso en el año 2016, a plazas de formación sanitaria especializada para Médicos, Farmacéuticos, Enfermeros y otros graduados/licenciados universitarios del ámbito de la Psicología, la Química, la Biología y la Física. Boletín Oficial del Estado, 224, 82031-82318. Recuperado de: http://boe.es/diario_boe/txt.php?id=BOE-A-201510053
Pacenza, M. I. y Silva Peralta, I. S. (2013). Análisis bibliométrico sobre responsabilidad social universitaria, Psychology, Society, \& Education, 5(2), 125-138. Recuperado de http://www.psye.org/articulos/Maria_Ines_Pacenza. pdf

Ramallo, M. (2015). La evaluación de la Responsabilidad Social Universitaria. Debate Universitario, 4(7), 25$37 . \quad$ Recuperado de http://ppct.caicyt.gov.ar/index.php/debateuniversitario/article/viewFile/6413/pdf

Real Decreto 2490/1998, de 20 de noviembre por el que se crea y regula el título oficial de Psicólogo Especialista en Psicología Clínica. Boletín Oficial del Estado, 288, 39538- 39542. Recuperado de https://www.boe.es/diario_boe/txt.php?id=BOE-A1998-27709

Real Decreto 183/2008, de 8 de febrero, por el que se determinan y clasifican las especialidades en ciencias de la salud y se desarrollan determinados aspectos del sistema de formación sanitaria especializada. Boletín Oficial del Estado, 45, 1002010035. Recuperado de https://www.boe.es/diario_boe/txt.php?id=BOE-A2008-3176

Real Decreto-ley 9/2011, de 19 de agosto, de medidas para la mejora de la calidad y cohesión del sistema nacional de salud, de contribución a la consolidación fiscal, y de elevación del importe máximo de los avales del Estado para 2011. Boletín Oficial del Estado, 200, 93143-93168. Recuperado de https://www.boe.es/diario_boe/txt.php?id=BOE-A2011-14021

Sanz Díaz-Palacios, J. A. (2015) La aportación académica al progreso: Hacia una auténtica responsabilidad social universitaria. Encuentros Multidisciplinares, 17(21), 7-18. Recuperado de http://www.encuentros-ultidisciplinares.org/revista51/jose_alberto_sanz_diaz-palacios.pdf 
Vallaeys, F. (2006). La Responsabilidad Social Universitaria. Lima: Pontificia Universidad Católica del Perú. World Health Organization (2014). Mental Health Atlas. WHO: Geneva.

\title{
PERFORMANCE OF GRADUATES IN THE INTERNSHIP IN CLINICAL PSYCHOLOGY ENTRANCE EXAMS
}

\author{
Eva EXPÓSITO-CASAS, ANA GONZÁlEZ-BENITO, \\ ESTHER LÓPEZ-MARTÍN Y ALEXIS MORENO-PULIDO
}

\section{EXTENDED SUMMARY}

\section{Introduction}

In Spain, university degree in Psychology is one of the most popular among prospective university students. As a result, Spanish public and private universities have increased the amount of places and the number of university graduates in Psychology. The access to the National Health System (NHS) is one professional possibility for these graduates. Applicants must pass an exam to become an Internship in Clinical Psychology at the NHS. This paper analyses the graduate's exam performance during the period 2013-2016, and also possible differences depending on size or ownership of their universities of origin.

\section{Method}

A non-experimental quantitative approach was used to address the research question by a descriptive approach of secondary data provided from the Ministry of Health, Social Services and Equality. Data are referred to the number of students who have passed the punctuation of screening in order to occupy places as an Internal Psychologist Resident (PIR) in 2013-2014, 2014-2015 and 2015-2016, as well as the total number of candidates submitted for the exam.

\section{Population and sample}

The study population consists of 10107 students who have been submitted to PIR selective test during the period under study. These students come from 49 universities which offer the degree in Psychology in Spain. During the preliminary analysis, 9 universities (8 privately owned and 1 public owned) were removed because, during the period considered, no student of these institutions had been presented to the selection tests for access to NHS. Thereby, the final subset of the target population available for study was comprised for 40 public and private universities, with two teaching modalities: traditional classroom or distance teaching.

\section{Variables}

Main variable is the score on the screening test, estimated from response of candidates to a multiplechoice test consisting of 225 items (plus 10 reserve questions) and limited to 5 hours of duration, characteristics common to the three calls analyzed (Decree SSI/1695/2013, 12 September; Decree SSI/1674/2014, 10 September; Decree SSI/1892/2015, 10 September).

The multiple choice test is considered surpassed for those candidates who obtain a particular value greater 
than or equal to $35 \%$ of arithmetic mean of ten best individual scores of the exercise, considering exclusively integers result of this operation (Decree SSI/1695/2013, 12 September; Decree SSI/1674/2014, 10 September; Decree SSI/1892/2015, 10 September).

The variables used to characterize the universities have been: Ownership (public/ private), size (big or small) and modality of teaching (traditional/ distance).

\section{Results}

\section{Evolution of the number of graduates in Psychology presented and that they overcome the selective tests for access to NHS}

During the period under review, there have been testing a total of 10107 undergraduates, 8563 of which have exceeded cut-off point. Given that applicants come from 40 Spanish universities, the average of graduates who faced and overcome these tests would be 253 and 214 people, respectively.

In any case, it is remarkable the huge variability in number of applicants who come from one or another institution of higher education. In this sense, we see how the number of subjects that each university has been submitted to the different calls is between 1 (2013-2014 and 2015-2016 calls) and 478 (call 2013-2015). In case of candidates who pass the tests, the observed maximum and minimum values are 0 (call 2013-2014) and 408 (2014-2015 and 2015-2016 calls).

In evolution analysis of subjects presented to the three calls, it can be observed how the possible differences in the number of graduates presented are not statistically significant (Chi-square Friedman $=3.279$, g.l. $=2, p=.194)$. On the contrary, there are significant differences in number of subjects who pass the tests of access to NHS in these calls (Chi-square Fried$\operatorname{man}=16.504$, g.1. $=2, p=.000)$. Thus, number of can- didates who exceeded the average grade of cut is lower in the call 2013-14 than in 2014-2015 calls (Wilcoxon $\mathrm{Z}=$ $3710, p=.000$.) and 2015-2016 (Wilcoxon $Z=-3.427$, $p=.289)$, no appreciable difference in the results of these last two calls (Wilcoxon $\mathrm{Z}=-1060, p=.289$ ).

\section{Percentage of individuals who pass the test access to NHS and analysis of differences depending on the size and ownership of universities}

Overall, the percentage of psychology graduates who passed the access test is more than $75 \%$ in the three calls. However, as was noted in previous section, variability of results obtained by graduates from different universities is important.

In all cases, the percentages distribution of approval obtained by public institutions is above the percentage distribution of private universities. In turn, in case of public universities the variability in the results obtained it is lower.

The analysis of the significance of observed differences, carried out through the Mann-Whitney U test, confirms the best results of students from public universities in 2013-2014 calls (Mann-Whitney $U=70.5$, $p=.016$ ) and 2014-2015 (Mann-Whitney $\mathrm{U}=76.0$, $p=.035)$, as well as throughout the observation period (Mann-Whitney $\mathrm{U}=82.0, p=.006$ ). Meanwhile, in the call 2015-2016, the percentage of approved subjects from both types of institutions does not differ significantly (Mann-Whitney $\mathrm{U}=119.0, p=.106$.).

The representation of the distribution of pass rates for different subgroups, reports a greater variability of the results obtained by small universities. In any case, the analysis of the differences in the percentages of subjects who pass the tests of access to NHS depending on the size of institutions, shows how the three calls considered: 20132015 (Mann-Whitney $\mathrm{U}=149.5, p=.838$ ), 2014-2015 (Mann-Whitney $\mathrm{U}=103.0, p=.177$ ) and 2015-2016 (Mann-Whitney $\mathrm{U}=172.0, p=.594$ ), these differences are not statistically significant. The superiority in the re- 
sults of a type of university over another is not apparent when considering the entire observation period (MannWhitney $\mathrm{U}=183.0, p=.817$ ).

\section{Performance and contribution of universities to NHS}

Focusing the analysis on the achievement obtained by these universities in the PIR selective test, we can appreciate how, of all the total subjects that have exceeded the cut-off mark in the various calls, a $78.34 \%$ come from face-to-face public universities, a $13.71 \%$ of the distance public university, $7.26 \%$ of all private universities face-to-face and finally, a $0.69 \%$ of the distance private university that is part of this study.

\section{Discussion}

The aim of this study was to analyze the performance of graduates in Psychology in selective testing access to specialized health training NHS, evaluating the educational and employment impact of universities of origin, which has allowed incorporating the approach to accountability a social dimension.

In recent years Psychology studies continue to be one of most demanded, the training offered (especially in private universities) has increased, and consequently, the number of graduates with such academic / professional profile.

An important way of professional development of these graduates is their specialization as a Clinical Psychologists; this specialization required overcome a test, set by the Management Act of the health professions (Law 44/2003 of 21 November). In light of the data from the last three calls (2013-2014, 2014-2015 and 20152016), we see how this is a highly demanded specialization, as the number of applicants in each of the calls was higher 3000 .

This paper provides empirical evidence about the great variability of the results obtained by graduates from different universities. In this regard the ownership of the institutions should be considered one of the variables that allow us to explain this differences. Thus, in the three calls analyzed, the percentage of students from public universities that pass the test was higher than the percentage of students from private schools. Such differences have proved to be statistically significant for 2013/2014 and 2014/2015 calls, as well as in the whole period. As for the size of the center, the differences have not been statistically significant, but is important to note the increased variability in the case of the results obtained by the smaller universities.

The study carried out, has allowed us identified the 12 universities that provide a higher number of students, superior to the mean and with better achievement on PIR test. These universities could be identified as a high social impact, because the quality of education that students receive, seems to translate into improved performance in test mentioned.

In relation to the analysis on the number of graduates who obtain a place in the training program PIR, the percentage of students from public universities that finally get place is greater than $90 \%$ in the three calls under study, reaching a $92.05 \%$ in the entire period. In light of the results, we can highlight the remarkable social contribution of university public centers to NHS.

In spite of the informative value of this study, is important to take into account some of the main limitations derived from available data. On the one hand, it would have been interesting to analyze the percentage of students that take the PIR test, in relation with the total number of graduates in those universities. By the other hand, it is not taken into consideration the post graduate training preparation of those candidates in order to prepare the exam and their possible influence in the results obtained. 